\title{
Comparison of outcomes between laparoscopic Vs. open liver resection for intermediate stage hepatocellular carcinoma
}

\author{
Avril David ${ }^{1,2}$, YoungRok Choi', Ho-Seong Han', Yoo-Seok Yoon ${ }^{1}$, Jai Young Cho' \\ 'Department of Surgery, Seoul National University College of Medicine, Seoul National University Bundang Hospital, Seongnam-si, \\ Gyeonggi-do 13620, South Korea. \\ ${ }^{2}$ Department of Surgery, Rizal Medical Center, Pasig City 1600, Philippines.
}

Correspondence to: Dr. YoungRok Choi, Department of Surgery, Seoul National University Bundang Hospital82, Gumi-ro 173 Beon-gil, Bundang-gu, Seongnam-si, Gyeonggi-do 13620, South Korea. E-mail: choiyoungrokr@gmail.com

How to cite this article: David A, Choi YR, Han HS, Yoon YS, Cho JY. Comparison of outcomes between laparoscopic vs. open liver resection for intermediate stage hepatocellular carcinoma. Hepatoma Res 2018;4:2. http://dx.doi.org/10.20517/2394-5079.2017.51

Received: 8 Dec 2017 First Decision: 11 Dec 2017 Revised: 18 Dec 2017 Accepted: 19 Dec 2017 Published: 18 Jan 2018

Science Editor: Kenneth S.H. Chok Copy Editor: Lu Liu Production Editor: Huan-Liang Wu

\begin{abstract}
Aim: The Barcelona Clinic Liver Score (BCLC) currently limits hepatic resection only for small, solitary tumors measuring $2-3 \mathrm{~cm}$ with no signs of portal hypertension (PHT) or macrovascular invasion. The aim of this study was to show the benefit of surgical resection, and to compare the peri-operative and long-term outcomes between laparoscopic liver resection (LLR) and open liver resection (OLR) for hepatocellular carcinoma (HCC) classified as intermediate stage $(B)$ under BCLC.
\end{abstract}

Methods: From 2004 to 2013, 49 patients staged as intermediate (BCLC B) and who underwent hepatic resection was included. These patients were divided into LLR or OLR. Demographics, tumor characteristics, recurrence rates and overall survival (OS) were compared between the 2 groups.

Results: Forty-nine patients were included and grouped into LLR $(n=28)$ and OLR $(n=21)$. The average tumor number was $2 \pm 1$ for both groups, while the mean tumor size was $4.4 \pm 1.7 \mathrm{~cm}$ and $5.3 \pm 2.6 \mathrm{~cm}$ for the LLR and OLR group, respectively. When compared with OLR, LLR had lower post-operative complications $(14.3 \% \mathrm{vs}$. $33.3 \%, P=0.114$ ), and a statistically significant shorter hospital stay than the OLR group ( 9 vs. 21 days, $P=0.023$ ). The LLR group also achieved a statistically significant difference in complete RO resection as compared with the OLR group $(P=0.016$ ). The OS and disease-free survival (DFS) at 1,3 and 5 years were comparable between LLR and OLR (OS: $89.1 \%$ vs. $76.2 \% ; 70.4 \%$ vs. $55.9 \% ; 58.6 \%$ vs. $43.5 \%, P=0.583 ;$ DFS: $59.3 \%$ vs. $51.0 \% ; 20.2 \%$ vs. $44.6 \% ; 16.2 \%$ vs. $37.2 \%, P=0.947$, respectively).

Conclusion: LLR showed comparable outcomes compared to OLR in the treatment of HCC staged BCLC B. Therefore, LLR as well as OLR can be considered in selective patients in the BCLC B group.

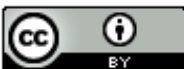

(C) The Author(s) 2018. Open Access This article is licensed under a Creative Commons Attribution 4.0 International License (https://creativecommons.org/licenses/by/4.0/), which permits unrestricted use sharing, adaptation, distribution and reproduction in any medium or format, for any purpose, even commercially, as long as you give appropriate credit to the original author(s) and the source, provide a link to the Creative Commons license, and indicate if changes were made.

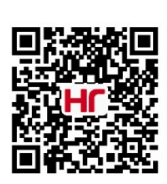


Keywords: Hepatocellular carcinoma, minimally invasive surgical procedures, laparoscopy

\section{INTRODUCTION}

Although incidence and mortality rates for cancer overall are declining, based on the 2017 National Comprehensive Cancer Network (NCCN) guidelines for hepatobiliary cancers, the incidence and mortality rates for liver cancer are increasing ${ }^{[1]}$. In particular, hepatocellular carcinoma (HCC) is the most common primary solid tumor in the world, and is ranked as the third leading cause of cancer-related death worldwide $^{[2]}$. Several staging systems have been developed in order to guide the management decisions in patients with HCC; among those, the Barcelona Clinic Liver Cancer (BCLC) Classification system has been approved and widely accepted in clinical practice ${ }^{[3]}$. According to the BCLC algorithm, curative treatment [including surgical resection, radiofrequency ablation (RFA) and transplantation] is recommended only for very early or early-stage (stage 0 -A) HCC, whereas palliative treatment is recommended for intermediate and advanced stage HCC (stage B-C ${ }^{[3]}$. In this regard, BCLC indication for curative resection is markedly limited. However, recent studies have shown that surgical resection can provide good outcomes for both short and long-term, despite the presence of portal hypertension, multi-nodular disease, large nodules $(>3 \mathrm{~cm})$, and those with macrovascular infiltration ${ }^{[4]}$. Particularly in the last decade, laparoscopic liver resection (LLR) may particularly be beneficial since it is less invasive, with significantly less post-operative complications, but with comparable oncologic outcomes to the open approach ${ }^{[5]}$.

The aim of this study is to show the benefit of surgical resection, and to compare the peri-operative and longterm outcomes, particularly in terms of recurrence and overall survival (OS), between LLR and open liver resection (OLR) for HCC classified as intermediate stage (B) under BCLC.

\section{METHODS}

\section{Study design and patient selection}

A retrospective review of the Electronic Medical Record database was done to identify all patients who underwent primary liver resection for HCC. From January 2004 to December 2013, a total of 1287 hepatectomies was performed at Seoul National University Bundang Hospital. Of these hepatectomies, 389 patients underwent liver resection for HCC. Among these patients, 49 patients staged as intermediate stage (BCLC B) were identified and selected. The study population included 2 comparable groups: OLR group composed of 21 patients, and the LLR group comprised of 28 patients.

\section{Preoperative evaluation}

A complete evaluation and surgical treatment for patients with HCC were discussed during multidisciplinary meetings. Liver function was evaluated using complete biochemical profiles and liver function tests, including indocyanine green (ICG) $)_{15}$ tests. Results were expressed as percentage of ICG retained 15 min $\left(\mathrm{ICG}_{15}\right)$ after injection. Resectability was decided by a multidisciplinary team approach.

\section{Liver volume measurement}

Triple-phase computed tomography (CT) was routinely used for preoperative imaging evaluation. The axial images were then loaded to a computer workstation where the semimanual Rapidia software (Infinitt Co., Ltd., Seoul, Korea) was installed. Cross-sectional areas of the liver on each transverse-slice image were obtained by manual tracing of the liver contour using a cursor, with free-curves drawn by experienced surgeons. Liver parenchymal volume was then generated automatically by summation of all the manually calculated areas of successive transverse-slice images ${ }^{[6]}$. The minimal amount of sufficient future liver remnant of $>30 \%$ was mandatory before surgery ${ }^{[7]}$.

\section{Surgical technique}

All liver resections were performed by a specialist hepatobiliary surgeon proficient in laparoscopic and open liver surgery. In general, the approach to both OLR and LLR was similar. Major anatomical resection 
was preferred for larger tumors or where tumor was in proximity to major vascular structures, requiring formal anatomical resection, otherwise non-anatomical resection was performed when adequate margins could be achieved. Standard vascular staping devices were used for both OLR and LLR when required, and combination of ultrasonic dissection using cavitron ultrasonic surgical aspirator and harmonic scalpel was used for parenchymal transection. Glissonian approach and individual approach were used to isolate and resect the hilar structures.

\section{Clinical outcomes}

Patient demographics, including age, gender, body mass index (BMI), previous abdominal surgery, RFA ortranscatheter arterial chemoembolization (TACE), and Child-Turcott-Pugh classification were recorded. Peri-operative outcomes included intraoperative and post-operative complication rate, severity of complications based on Clavien-Dindo classification, type of hepatic resection, resection margin status, estimated blood loss, length of stay, total operative time (incision to closure time), and amount of transfusion. Histological analysis of resected HCC specimens was also assessed, including the Edmondson histological grade, PT staging, microvascular invasion, tumor number, and maximal tumor diameter.

\section{Follow-up, survival and recurrence}

After resection, patients were followed up 1 month after surgery, then every 3 months in the first 2 postoperative years and then at 6-month intervals for post-operative years 3 to 5 , using serum $\alpha$-feto-protein (AFP), with multi-phase contrast enhanced CT or magnetic resonance imagin (MRI), or Gadoxetate disodium (Gd-EOB-DTPA) enhanced MRI of the liver.

The OS was calculated from the day of surgery until the day of death or last contact. The recurrence-free survival of patients who recurred was defined as the time from the day of surgery to the day of imaging study that confirmed tumor recurrence.

\section{Statistical analysis}

Descriptive statistics were reported as a mean with standard deviation for continuous variables, and as a number and percentage for discrete variables. OS and recurrence-free survival were calculated by the Kaplan-Meier method and differences were compared by the log-rank test. Statistical significance was defined as $P<0.05$. Statistical analysis was carried out using SPSS software (version 20; SPSS, Chicago, IL, USA).

\section{RESULTS}

\section{Clinicopathologic characteristics of patients with BCLC stage B according to treatment group}

Demographics and peri-operative outcomes according to treatment group are shown in Table 1. Six out of 28 patients required conversion from a laparoscopic to open resection. There was no statistical difference between these groups in terms of age, gender, BMI, history of previous abdominal surgery, biochemistry profiles (albumin, bilirubin, prothrombin, AST and ALT levels, platelet), and preoperative AFP levels.

Majority of resections in the LLR group were minor resections $(75.0 \%$ vs. 38.1\%, $P=0.009)$. The LLR group had longer mean operation time (350 vs. $339 \mathrm{~min}, P=0.066)$ and higher estimated blood loss (1707 vs. $1055 \mathrm{~mL}$, $P=0.039$ ). However, LLR group was able to achieve Ro resection in all resections, compared to $81.0 \%$ in the OLR group.

\section{Histological findings}

There were a greater proportion of cirrhotic patients in the LLR and OLR groups (67.9\% vs. 42.9\%, respectively, $P=0.080$ ) [Table 2]. Although the OLR group had significantly greater mean tumor diameter $(P=0.048)$, there was no significant difference between the number of tumors removed in both treatment groups $(P=0.074)$. There was also no statistically significant difference of resection margin, presence of microvascular invasion and Edmondson-Steiner grading between LLR and OLR groups $(P=0.649,0.740$ and 
Table 1. Basic characteristics of patients with BCLC stage B according to treatment group

\begin{tabular}{|c|c|c|c|}
\hline & OLR $(n=21)$ & $\operatorname{LLR}(n=28)$ & $\boldsymbol{P}$ \\
\hline Age (years) & $63 \pm 12$ & $58 \pm 11$ & 0.080 \\
\hline Sex (\% male) & $17(81 \%)$ & $18(64 \%)$ & 0.201 \\
\hline $\mathrm{BMI}\left(\mathrm{Kg} / \mathrm{m}^{2}\right)$ & $23.0 \pm 3.2$ & $24.8 \pm 3.7$ & 0.094 \\
\hline Previous abdominal surgery & $4(19.0 \%)$ & $1(3.6 \%)$ & 0.077 \\
\hline Previous TACE & $11(52.4 \%)$ & $6(21.4 \%)$ & 0.024 \\
\hline Previous RFA & $3(14.3 \%)$ & $0(0 \%)$ & 0.039 \\
\hline Child-Turcott-Pugh classification & & & 0.122 \\
\hline A & $21(100 \%)$ & $25(89.3 \%)$ & \\
\hline$B$ & 0 & $3(10.7 \%)$ & \\
\hline Albumin $(\mathrm{g} / \mathrm{dL})$ & $4.0 \pm 0.4$ & $4.1 \pm 0.4$ & 0.918 \\
\hline Total bilirubin (mg/dL) & $0.7 \pm 0.2$ & $0.8 \pm 0.4$ & 0.457 \\
\hline Prothrombin time (INR) & $1.1 \pm 0.1$ & $1.1 \pm 0.1$ & 0.110 \\
\hline AST $(U / L)$ & $37.1 \pm 22.9$ & $39.2 \pm 19.7$ & 0.742 \\
\hline $\operatorname{ALT}(U / L)$ & $32.1 \pm 17.7$ & $38.9 \pm 19.8$ & 0.218 \\
\hline Platelet (x 1000/mcL) & $162.1 \pm 82.5$ & $139.4 \pm 57.3$ & 0.289 \\
\hline Viral disease & & & 0.727 \\
\hline $\mathrm{HCV}$ & $14(66.7 \%)$ & $20(71.4 \%)$ & \\
\hline HBV & $2(9.5 \%)$ & $4(14.3 \%)$ & \\
\hline Both positive & 0 & 0 & \\
\hline Both negative & $5(23.8 \%)$ & $4(14.3 \%)$ & \\
\hline ICGR15 (\%) & $10.0 \pm 4.2$ & $9.9 \pm 7.9$ & 0.937 \\
\hline $\operatorname{AFP}(n g / m L)$ & $1191.4 \pm 4750.7$ & $539.2 \pm 1491.3$ & 0.550 \\
\hline Type of hepatic resection & & & 0.009 \\
\hline Minor & $8(38.1 \%)$ & $21(75.0 \%)$ & \\
\hline Major & $13(61.9 \%)$ & $7(25.0 \%)$ & \\
\hline Resection margin $(\mathrm{cm})$ & $1.7 \pm 2.4$ & $1.4 \pm 1.5$ & 0.610 \\
\hline RO & $17(81.0 \%)$ & $28(100.0 \%)$ & 0.017 \\
\hline $\mathrm{R} 1$ & $4(19.0 \%)$ & 0 & \\
\hline Operation time (min) & $339.0 \pm 90.9$ & $350.3 \pm 171.2$ & 0.066 \\
\hline Estimated blood loss ( $\mathrm{mL}$ ) & $1055.3 \pm 889.6$ & $1707.5 \pm 3294.7$ & 0.039 \\
\hline Transfusion done & $9(42.9 \%)$ & $12(42.9 \%)$ & 1.000 \\
\hline $\mathrm{RBC}$ & $2.7 \pm 1.8$ & $6.3 \pm 5.8$ & 0.022 \\
\hline FFP & $0.8 \pm 1.6$ & $2.1 \pm 2.4$ & 0.056 \\
\hline
\end{tabular}

LLR: Iaparoscopic liver resection; OLR: open liver resection; BMI: body mass index; TACE:transarterial chemoembolization; RFA: radiofrequency ablation; AST: aspartate aminotransferase; ALT: alanine aminotransferase; HBV: hepatitis B virus; HCV: hepatitis C virus; ICGR15:indocyanine green retention test at 15 min; AFP: alpha-fetoprotein; PRBC: packed RBC; red blood cell, FFP: fresh frozen plasma. Values are expressed as mean \pm standard deviation or number (percent).

0.425 respectively). In the pathologic TNM stage, the T stage was different between two groups $(P=0.031)$.

\section{Postoperative complications, hospital stay and recurrence}

As seen in Table 3, the LLR group had a higher rate of intraoperative complications than the OLR group (17.9\% vs. $14.3 \% ; P=0.738)$, but a lower rate of postoperative complications $(14.3 \%$ vs. $33.3 \%$; $P=0.114)$. There was no significant difference in overall serious postoperative complications between the LLR and OLR groups.

Patients in the LLR group had a significantly shorter postoperative hospital stay (median = 9 days; range: 4-30) than the OLR group (median $=21$ days; range: $7-147, P=0.023$ ).

In terms of recurrence, LLR had similar recurrence rates with OLR $(78.6 \% v s .71 .4 \%, P=0.565)$ during the follow-up of $63 \pm 40$ months and $48 \pm 43$ months in LLR and OLR respectively. For both LLR and OLR, majority of the recurrences were intrahepatic recurrences ( $77.3 \%$ vs. $93.3 \%$, respectively).

\section{Overall survival and disease-free survival}

The OS at 1,3 and 5 years for LLR was $89.1 \%, 70.4 \%$ and $58.6 \%$, respectively; and $76.2 \%, 55.9 \%$ and $43.5 \%$, respectively for OLR. OS for both groups was $\operatorname{similar}(P=0.583)$ and there was also no statistically 
Table 2. Pathologic findings in patients according to treatment group

\begin{tabular}{llcc}
\hline & OLR $(\boldsymbol{n}=\mathbf{2 1})$ & LLR $(\boldsymbol{n}=\mathbf{2 8})$ & $\boldsymbol{P}$ \\
\hline Greatest tumor size (cm; mean 4 SD) & $5.3 \pm 2.6$ & $4.4 \pm 1.7$ & 0.048 \\
Tumor number & $2.0 \pm 1.2$ & $1.5 \pm 0.7$ & 0.074 \\
Margin distance (cm; mean 4 SD) & $1.7 \pm 2.4$ & $1.4 \pm 1.5$ & 0.649 \\
ES tumor grade (I/II/III/IV) & $0: 8: 12: 1: 0$ & $1: 12: 10: 4: 1$ & 0.425 \\
PT stage & & & 0.031 \\
T1 & 2 & 9 & \\
T2 & 9 & 15 & \\
T3 & 9 & 2 & \\
T4 & 1 & 1 & \\
Cirrhosis present & $9(42.9 \%)$ & $19(67.9 \%)$ & 0.080 \\
Microvascular invasion & $10(47.6 \%)$ & $12(42.9 \%)$ & 0.740 \\
\hline
\end{tabular}

LLR: laparoscopic liver resection; OLR: open liver resection; ES: Edmondson-Steiner. Values are expressed as mean \pm standard deviation or number (percent).

Table 3. Complications according to treatment group

\begin{tabular}{llll}
\hline & OLR (n= 21) & LLR (n= 28) & P \\
\hline Intraoperative & $3(14.3 \%)$ & $5(17.9 \%)$ & 0.738 \\
Postoperative & $7(33.3 \%)$ & $4(14.3 \%)$ & 0.114 \\
Clavien-Dindo classification & & & 0.256 \\
Grade I & 2 & 0 & 0 \\
Grade II & 0 & 3 & 1 \\
Grade IIIa & 3 & 0 & \\
Grade IIIb & 2 & $4(14.3 \%)$ & 0.237 \\
Grade IVa/IVb & 0 & & \\
Postoperative serious complications & $5(23.8 \%)$ & $9.2 \pm 4.9(4-30)$ & 0.023 \\
(Clavien-Dindo Grade III or higher) & & & \\
Hospital stay (days; mean \pm SD: range) & $20.6 \pm 30.1(7-147)$ & & \\
\hline
\end{tabular}

LLR: laparoscopic liver resection; OLR: open liver resection

significant difference in terms of DFS between the groups $(P=0.947)$. The $1-, 3-$ and 5 -year DFS was 59.3\%, $20.2 \%$ and $16.2 \%$ for the LLR group and $51.0 \%, 44.6 \%$ and $37.2 \%$ for OLR groups, respectively [Figure 1].

\section{DISCUSSION}

HCC is widely endemic, with $80 \%$ of new cases worldwide expected to develop in Asia alone ${ }^{[8]}$. The management of HCC is complex, due to the presence of two disease processes: the primary malignancy and the underlying liver pathology that accompanies HCC. Thus a reliable management algorithm to guide therapeutic decisions in these patients is needed.

Currently, the BCLC classification system is one of the most widely recognized and approved staging systems for HCC, since it considers multiple factors such as tumor stage and function, patient's performance status, as well as cancer-related symptoms ${ }^{[9]}$. According to BCLC, intermediate stage (BCLC-B) patients are asymptomatic (PS score 0), with large multi-nodular tumors but without macrovascular invasion or extrahepatic spread ${ }^{[8]}$. The estimated 3-year survival for patients with untreated stage B HCC ranges from $8 \%$ to $50 \%$; given the invasiveness of surgery, palliation with TACE is therefore recommended for these subset of patients ${ }^{[\rho]}$. However, TACE cannot induce complete tumor necrosis especially in large tumors, with reported response rates in literature as low as $2 \%{ }^{[8,10]}$.

Liver resection, based on the BCLC system, is usually reserved for patients with small, single tumors, with absence of portal hypertension or hyperbilirubinemia ${ }^{[9]}$. Recent data has however, supported the benefit of surgical resection in terms of short- and long-term oncologic outcomes despite the presence of large, multinodular nodules and macrovascular invasion ${ }^{[4]}$. In a recent study done by Kim et al ${ }^{[11]}$, overall median 

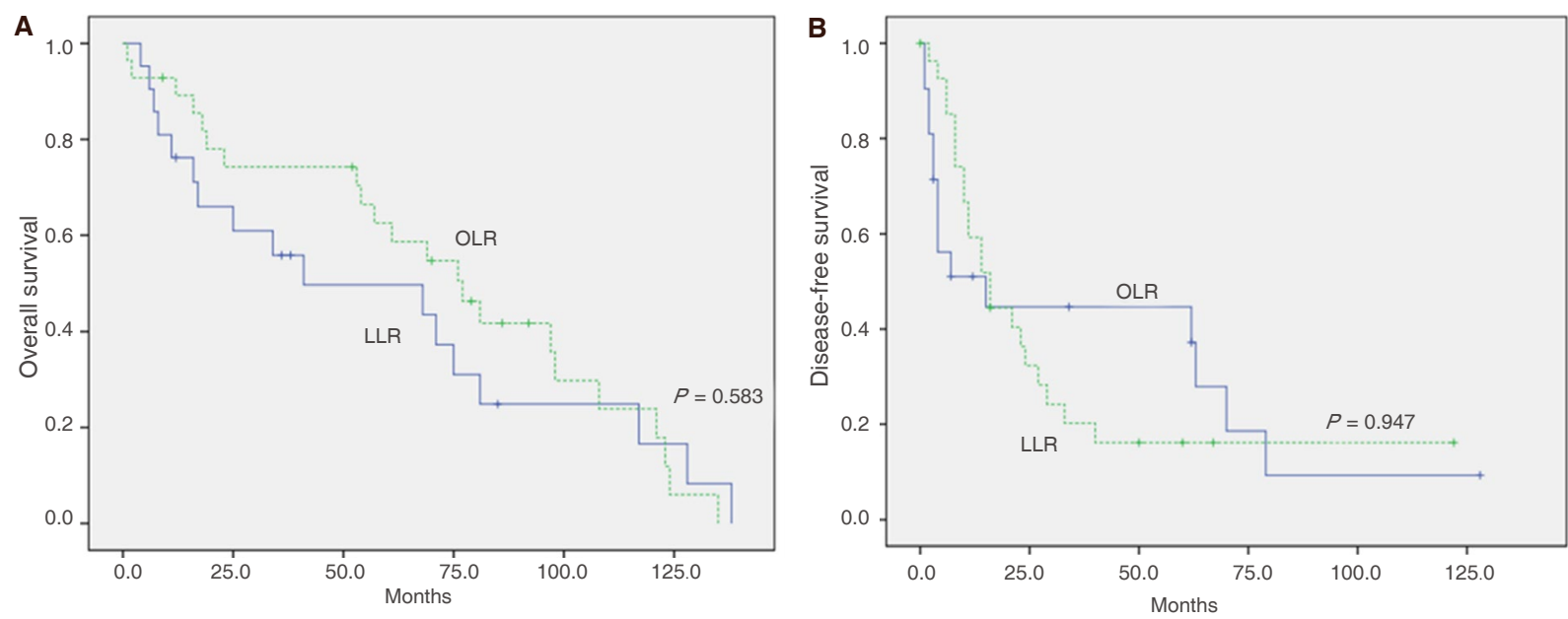

Figure 1. Overall survival andrecurrence-free survival for laparoscopic and open liver resection for intermediate stage BCLC-B hepatocellular carcinoma. A: Overall survival. B: Recurrence-free survival for laparoscopic and open liver resection for intermediate stage BCLC-B hepatocellular carcinoma. Solid line: open liver resection (blue line); dot line: lap liver resection (green line). The OS at 1, 3 and 5 years for LLR was $89.1 \%, 70.4 \%$ and $58.6 \%$, respectively; and $76.2 \%, 55.9 \%$ and $43.5 \%$, respectively for OLR $(P=0.583)$. The $1-, 3-$ and 5-year DFS was 59.3\%, 20.2\% and $16.2 \%$ for the LLR group and $51.0 \%, 44.6 \%$ and $37.2 \%$ for OLR groups, respectively $(P=0.947)$. LLR:Iaparoscopic liver resection; OLR: open liver resection; BCLC: Barcelona Clinic Liver Score

survival was higher in patients who received surgical resection compared to those who received non-surgical therapy (50.9 vs. 22.1 months), with an overall 5-year survival of 63\% in the resection group. This is in part due to recent advances in surgical devices and techniques and improved perioperative care, with some highvolume centers reporting surgical mortality rates as low as $0.8 \%{ }^{[12]}$.

However, because of the underlying liver disease concomitant with HCC, patients undergoing open liver resection are still at a high risk of developing significant postoperative complications ${ }^{[2]}$. LLR, since its inception in 1993, is currently being considered as a feasible, safe and less invasive alternative to open surgery in the case of malignant hepatic tumors ${ }^{[13]}$. Particularly, the benefit of LLR is more pronounced in this population of cirrhotic patients, despite initial studies that considered it a contraindication. The minimally invasiveness of LLR may decrease the risk of peri-operative complications and mortality ${ }^{[9]}$, as evidenced by our present study wherein the LLR group was associated with a lower rate of post-operative complications. In terms of mortality, there was no observed 90-day post-operative mortality in both the LLR and OLR groups. Previous studies have shown longer operative times for laparoscopic surgery ${ }^{[13]}$, and this is also consistent with our study because most of the cases included were done during the early phase of LLR.

Adequacy of resection margin, which was initially a limitation for laparoscopic surgery due to the inability of direct palpation of tumor, has been overcome by the use of intraoperative Doppler ultrasonography, and has greatly facilitated the achievement of good oncologic outcomes. Indeed as shown in our present study, tumor-free margins on pathological examination were similar between the LLR and OLR group, and this is consistent with results of previous studies ${ }^{[13]}$.

The high propensity of HCC for recurrence after resection is well-documented, with reported recurrence rates ranging from $70 \%$ to $100 \%$; among several factors, number of tumors was noted to be the most significant predictor. Indeed, previous data have shown that the 5-year DFS rates after surgical resection for multiple HCCs ranges from 0 to $26 \%{ }^{[12]}$. This is consistent with our present study, which revealed comparable 5-year DFS rates for both the LLR and OLR group at $16.2 \%$ and $37.2 \%$, respectively, despite the presence of multiple tumors for both treatment groups. Also, our study showed that liver resection was associated with 5 -year OS rates of almost $60 \%$ for the LLR group and $44 \%$ for the OLR group, despite the presence of multiple, large tumors. 
One of the major limitations of this study is its retrospective nature and small sample size with difference in basic characteristics in both groups. However, our 5 -year OS rate of $60 \%$ and $44 \%$ for LLR and OLR, respectively, is satisfactory, and may justify the expansion of indications for surgical resection in the case of multiple, large HCC, if the liver functions remains at Child-Pugh class A.

In conclusion, this retrospective study demonstrates that LLR and OLR have comparable OS and DFS rates for BCLC-B HCC patients with multiple or large tumors. Particularly, decreased postoperative complications and shorter hospital stay, with successful achievement of adequate resection margins, was observed in the LLR group. It was demonstrated that good oncologic and perioperative outcomes can be achieved with LLR for HCC.

\section{DECLARATIONS}

\section{Authors' contributions}

Collecting and analyzing data, writing the manuscript: David A

Design this study, analyzing data, finalizing the manuscript: Choi YR

Analyzing data and drafting the manuscript: Han HS, Yoon YS, Cho JY

\section{Data source and availability}

Data and survey materials are available upon request from the corresponding author.

\section{Financial support and sponsorship}

None.

\section{Conflicts of interest}

There are no conflicts of interest.

\section{Patient consent}

Agreement exemption allowed by the Institutional Review Board (IRB) of Seoul National University Bundang Hospital (SNUBH).

\section{Ethics approval}

This retrospective, observational study was approved by the Institutional Review Board (IRB) of Seoul National University BundangHospital (SNUBH, B-1801-442-108).

\section{Copyright}

(c) The Author(s) 2018.

\section{REFERENCE}

1. National Comprehensive Cancer Network. Clinical practice guidelines in oncology: hepatobiliary cancers. Version 4.2017.

2. Lee JJ, Conneely JB, Smoot RL, Gallinger S, Greig PD, Moulton CA, Wei A, McGilvray I, Cleary SP. Laparoscopic vs open liver resection for hepatocellular carcinoma at a North American Center: a 2-to-1 matched pair analysis. HPB (Oxford) 2015;17:304-10.

3. Bruix J, Sherman M. Management of hepatocellular carcinoma: an update. Hepatology 2011;53:1020-2.

4. Garancini M, Pinotti E, Nespoli S, Romano F, Gianotti L, Giardini V. Hepatic resection beyond barcelona clinic liver cancer indication; when and how. World J Hepatol 2016;8:513-19.

5. Cipriani F, Fantini C, Ratti F, Lauro R, Tranchart H, Halls M, Scuderi V, Barkhatov L, Edwin B, Troisi RI, Dagher I, Reggiani P, Belli G, Aldrighetti L, Abu Hilal M. Laparoscopic liver resections for hepatocellular carcinoma: can we extend the surgical indication in cirrhotic patients? Surg Endosc 2017; doi: 10.1007/s00464-017-5711-x.

6. Mussin N, Sumo M, Lee KW, Choi YR, Choi JY, Ahn SW, Yoon KC, Kim HS, Hong SK, Yi NJ, Suh KS. The correlation between preoperative volumetry and real graft weight: comparison of two volumetry programs. Ann Surg Treat Res 2017;92:214-20.

7. Nugroho A, Kim OK, Lee KW, Song S, Kim H, Hong SK, Yoon KC, Kim HS, Choi YR, Lee HW, Yi NJ, Suh KS. Evaluation of donor workups and exclusions in a single-center experience of living donor liver transplantation. Liver Transpl 2017;23:614-24.

8. Han KH, Kudo M, Ye SL, Choi JY, Poon RT, Seong J, Park JW, Ichida T, Chung JW, Chow P, Cheng AL. Asian consensus workshop report: expert consensus guidelines for the management of intermediate and advanced hepatocellular carcinoma in Asia. Oncology 2011;81:158-64. 
9. Torzilli G, Donadon M, Marconi M, Palmisano A, Del Fabbro D, Spinelli A, Botea F, Montorsi M. Hepatectomy for stage B and C hepatocellular carcinoma in the Barcelona Clinic Liver Cancer classification. Arch Surg 2008;143:1082-90.

10. Kanazawa A, Tsukamoto T, Shimizu S, Kodai S, Yamazoe S, Yamamoto S, Kubo S. Impact of laparoscopic liver resection for hepatocellular carcinoma with F4 liver cirrhosis. Surg Endosoc 2013;27:2592-97.

11. Kim H, Ahn SW, Hong SK, Yoon KC, Kim HS, Choi YR, Lee HW, Yi NJ, Lee KW, Suh KS. Survival benefit of liver resection for Barcelona Clinic Liver Cancer stage B hepatocellular carcinoma. Br J Surg 2017;104:1045-52.

12. Ishizawa T, Hasegawa K, Aoki T, Takahashi M, Inoue Y, Sano K, Imamura H, Sugawara Y, Kokudo N, Makuuchi M. Neither multiple tumors nor portal hypertension are surgical contraindications for hepatocellular carcinoma. Gastroenterology 2008;134:1908-16.

13. Memeo R, de Angelis N, Compagnon P, Salloum, C, Cherqui D, Laurent A, Azoulay D. Laparoscopic vs open liver resection for hepatocellular carcinoma of cirrhotic liver: a case-control study. World J Surg 2014;38:2919-26. 